Instituto Internacional de Investigación y Desarrollo Tecnológico Educativo INDTEC, C.A.

DOI: https://doi.org/10.29394/Scientific.issn.2542-2987.2021.6.22.11.210-230

OAl-PMH: http://www.indteca.com/ojs/index.php/Revista Scientific/oai

Artículo Original / Original Article

\title{
Desarrollo sostenible: Casos empresas exportadoras de camarón en la provincia de EI Oro
}

Autores: Aylin Madelaine Honores Gómez Universidad Técnica de Machala, UTMACH ahonores3@utmachala.edu.ec Machala, Ecuador https://orcid.org/0000-0002-3867-3943

Byron Alexander Chavarría Sangurima Universidad Técnica de Machala, UTMACH bchavarri1@utmachala.edu.ec Machala, Ecuador https://orcid.org/0000-0003-3664-0002

Sandra Sayonara Solórzano Solórzano Universidad Técnica de Machala, UTMACH ssolorzano@utmachala.edu.ec Machala, Ecuador https://orcid.org/0000-0001-6294-7396

\section{Resumen}

El desarrollo sostenible y las empresas juegan un papel primordial al buscar satisfacer las necesidades presentes sin comprometer las generaciones futuras. Una organización es sustentable cuando logra crear un valor económico, social y medioambiental entre todos los stakeholders que la conforman. Con el objetivo de evaluar el desempeño en desarrollo sostenible en las empresas exportadoras de camarón de la provincia de El Oro, Ecuador, para así conocer como contribuyen al desarrollo local sustentable, se realizó una investigación de tipo descriptiva de corte transversal y de campo empleando un paradigma cualitativa-cuantitativa. Se utilizaron los métodos científicos, teóricos y empíricos, que permitieron exponer la situación que poseen las empresas exportadoras de camarón de la provincia de El Oro además se evidencio un desempeño favorable en cuanto a su desarrollo económico, ambiental y social, es decir sus estrategias están enfocadas al desarrollo de nuevas tecnologías y gestiones ambientales como certificaciones socialmente responsables con sus empleados, clientes, proveedores y comunidad haciendo sus procesos más eficientes y eficaces. Finalmente se puntualiza con los beneficios que poseen las empresas al implementar estas estrategias.

Palabras clave: desarrollo sostenible; empresas; exportación; stakeholders. Código de clasificación internacional: 5304.04 - Comercio exterior.

Cómo citar este artículo:

Honores, A., Chavarría, B., \& Solórzano, S. (2021). Desarrollo sostenible: Casos empresas exportadoras de camarón en la provincia de El Oro. Revista Scientific, 6(22), 210-230. Recuperado de: https://doi.org/10.29394/Scientific.issn.2542-2987.2021.6.22.11.210-230

Fecha de Recepción: 17-10-2021
Fecha de Aceptación: 02-10-2021
Fecha de Publicación: 05-11-2021 
OAI-PMH: http://www.indteca.com/ojs/index.php/Revista Scientific/oai

Artículo Original / Original Article

\title{
Sustainable development: Cases of shrimp exporting companies in the province of EI Oro
}

\begin{abstract}
Sustainable development and companies play a fundamental role in seeking to satisfy present needs without compromising future generations. An organization is sustainable when it manages to create economic, social and environmental value among all the stakeholders that comprise it. In order to evaluate the performance in sustainable development in shrimp exporting companies in the province of El Oro, Ecuador, in order to know how they contribute to sustainable local development, a descriptive cross-sectional and field investigation was carried out using a qualitative-quantitative paradigm. Scientific, theoretical and empirical methods were used, which allowed to expose the situation of shrimp exporting companies in the province of El Oro, in addition, a favorable performance was evidenced in terms of their economic, environmental and social development, that is, their strategies are focused on the development of new technologies and environmental management such as socially responsible certifications with its employees, customers, suppliers and the community, making its processes more efficient and effective. Finally, it is specified with the benefits that companies have when implementing these strategies.
\end{abstract}

Keywords: sustainable development; business; export; stakeholders. International classification code: 5304.04 - Foreign trade.

\section{How to cite this article:}

Honores, A., Chavarría, B., \& Solórzano Solórzano, S. (2021). Sustainable development: Cases of shrimp exporting companies in the province of El Oro. Revista Scientific, 6(22), 210-230. Recovered from: https://doi.org/10.29394/Scientific.issn.2542-2987.2021.6.22.11.210-230

Date Received: 07-10-2021
Date Acceptance: 02-10-2021
Date Publication: 05-11-2021 


\section{Introducción}

La globalización para la Oficina Internacional del Trabajo (OIT, 2007a): fomenta nuevos desafíos y oportunidades entre el sector privado y las economías nacionales, al confrontar a las empresas a las dificultades económicas, sociales y ambientales, generando una nueva y mejor relación entre las organizaciones y el desarrollo.

Los constantes cambios en el mercado internacional generan grandes oportunidades de negocios, los mismo que son aprovechados por las organizaciones, así mismo pueden ser percibidos como posibles amenazas para otros. Acorde a Mora-Córdova, Lituma-Loja y González-Illescas (2020): las empresas exportadoras de camarón buscan mantener un constante desarrollo que pueda adaptarse a las condiciones que estas deben enfrentar y con ello la búsqueda de ventajas competitivas.

La empresa sostenible se relaciona con el concepto de desarrollo sostenible, en este sentido, para la Organización Mundial del Comercio (OMC, 2011): se debe "asegurar que satisfaga las necesidades del presente sin comprometer la capacidad de las futuras generaciones para satisfacer las propias" (pág. 2). Según la Oficina Internacional del Trabajo (OIT, 2007b): el desarrollo sostenible integra el crecimiento económico, el progreso social y los aspectos medioambientales, creando así los tres pilares fundamentales que lo conforman.

Al respecto, Zarta (2018): complementa sobre la sustentabilidad siendo clave para el desarrollo del hombre y su entorno, la misma que debe considerarse como disciplina integradora que conlleve a una revolución global con el medio ambiente y la humanidad.

Mencionando a Manfredi y González (2017): una estrategia es un mecanismo necesario que permite resolver dificultades y alcanzar mejores resultados en una negociación. Las estrategias en desarrollo sostenible son consideradas como herramientas o mecanismos integradores que permiten 
compatibilizar los diferentes objetivos que tienen las empresas, para cumplir sus metas de manera eficaz, conjuntamente con los sectores primordiales de lo social, económico y medioambiental.

Interpretando a Contreras-Pacheco, Talero-Sarmiento y EscobarRodríguez (2020): las empresas socialmente responsables buscan crear un escenario en donde propietarios, empleados, comunidades, sociedades, controlen el impacto medioambiental y social derivado del comportamiento de cada una de ellas.

Las reglas de la sociedad han cambiado, tal como lo explica el Banco Bilbao Vizcaya Argentaria (BBVA, 2014): la forma de regir los negocios, a tal punto que las empresas ya no consideran el generar dinero, para los accionistas, como prioridad para la creación de valor en una organización, sino que, para alcanzar el éxito en una empresa se debe entablar una relación sólida y de confianza con sus partes interesadas, sociedad y entorno

Los stakeholders conceptualizados por Freeman y McVea (2001): los consideran como grupos o individuos que pueden ser afectados por las decisiones de la organización. La gestión de los stakeholders debe considerar el trabajo conjunto, de forma que se considere simultáneamente todas sus partes interesadas, buscando así el cumplimiento de los objetivos propuestos por la empresa.

Cabe mencionar que para el Grupo de Desarrollo Sostenible de las Naciones Unidas para América Latina y el Caribe, Panamá (UNSDG, 2018): la transición de las empresas hacia el desarrollo sostenible debe ser apoyado al uso de herramientas tecnológicas y mecanismos enfocados a la legislación de cada país, en ser de educación, políticas y leyes, alianzas estratégicas y desarrollo de la sociedad, con la finalidad de lograr el éxito de cada una de ellas.

Los modelos de negocio sostenibles como indica Schneider (2019a): priorizan la transparencia en gestión de riesgos y oportunidades tanto 
económicas, sociales y ambientales, lo que impulsa actualmente a las empresas que integren, en sus estrategias, los tres elementos que conforman la sostenibilidad empresarial.

El artículo se realizó en base a información obtenida del centro de investigación de la Universidad Técnica de Machala titulado percepción de los consumidores de las acciones de desarrollo sostenible empleadas por las organizaciones del sector minorista en El Oro.

Para alcanzar el objetivo principal de la investigación, se realiza un estudio de tipo descriptivo de corte transversal y de campo empleando un paradigma cualitativo y cuantitativo, ya que los datos obtenidos dependen de la interpretación y subjetividad del investigador. Se emplea los métodos teóricos analítico-sintético e inductivo-deductivo para caracterizar, identificar y valorar la situación actual sobre desarrollo sostenible en las empresas exportadoras de camarón de la provincia de El Oro.

Se utiliza el método empírico de la encuesta, útil para recabar información sobre el desarrollo sostenible, aplicada a un total de siete empresas registradas como exportadoras de camarón en la provincia de El Oro en el año 2021, siendo la totalidad de la población que la investigación utiliza.

El estudio expone como principal resultado el desempeño que poseen las empresas exportadoras de camarón de la provincia de El Oro, respecto a su desarrollo sostenible, además de una recopilación de información de casos similares que permita apreciar la relación con la investigación. Finalmente, en la conclusión se puntualiza los beneficios de adoptar estrategias que impulsen el desarrollo sostenible en las organizaciones.

La investigación tiene como objetivo principal evaluar el desempeño sobre desarrollo sostenible en las empresas exportadoras de camarón de la provincia de El Oro, Ecuador, mediante un estudio de sus variables para así conocer como contribuyen al desarrollo local sustentable. 


\section{Metodología (Materiales y métodos)}

Para esta investigación se realizó un estudio de tipo descriptivo de corte transversal y de campo empleando un paradigma cualitativo y cuantitativo. Se utilizaron los métodos teóricos analítico-sintético e inductivo y deductivo para evaluar y valorar la situación actual sobre el desarrollo sostenible en las empresas exportadoras de camarón de la provincia de El Oro en el año 2021.

Para identificar las características del desarrollo sostenible y casos de estudios relacionados a la investigación, se trabajó con una bitácora conformada por artículos científicos indexados en bases de datos como SciELO, Scopus, Redalyc y Latindex, los mismos que son parte del proyecto Percepción de los consumidores de las acciones de desarrollo sostenible empleadas por las organizaciones del sector minorista en El Oro, perteneciente al centro de investigación de la Universidad Técnica de Machala.

Los métodos empíricos se determinaron a partir del objetivo de la investigación y su relación con los objetivos específicos, por tal, para recabar información útil sobre la situación de desarrollo sostenible, se aplicó el método no experimental de tipo descriptivo utilizando la técnica de la encuesta y su instrumento el cuestionario.

Para obtener la población de estudio se tomó como referencia la base de datos de empresas exportadoras de la provincia de El Oro, siendo un total de 84 empresas desglosadas en 10 sectores: Bananero, cacaotero, cafetero, camaronero, textil, forestal, alimentos procesados, artesanal, fruta no tradicional y servicios. Para delimitar la población, la investigación se enfocó en el sector productivo camaronero debido a la influencia en la economía ecuatoriana al ser el segundo rubro de exportación no petrolera más importante del país. Se registró un total de siete empresas en la provincia, por tanto, debido al número limitado se trabajó con el total de la población.

El instrumento de la encuesta estuvo estructurado por un total de 16 preguntas de tipo dicotómicas de única respuesta (SI o NO) las cuales fueron 
divididas en cuatro variables de estudio (conocimiento, desarrollo social, desarrollo medio ambiental y desarrollo económico) lo que permitió medir la situación sobre el desarrollo sostenible en las empresas camaroneras.

Para interpretar los resultados y conseguir el promedio de cada variable de estudio (social, medio ambiental y económico), se realizó una suma de todos los datos obtenidos dividiéndose para el número total de criterios.

\section{Resultados (análisis e interpretación de los resultados)}

Para obtener los resultados de la investigación se establecieron diversos criterios para calificar la realidad de la población, enfatizando los aspectos menos aplicados por las organizaciones, para así dar a conocer finalmente la situación actual en desarrollo sostenible de las organizaciones establecidas como objeto de estudio.

El resultado de cada variable: social, económico y medioambiental, están basados en las preguntas de la encuesta realizada a la población de estudio. Cada criterio establecido fue tabulado porcentualmente por el número total de respuestas obtenidas de cada criterio de evaluación en cada empresa, obteniendo así una valoración porcentual general de toda la población.

La primera variable de estudio indago la responsabilidad de las empresas desde el punto de vista social. Con un total de ocho criterios basados en su relación con sus empleados, proveedores, clientes y comunidad, evidenciándose lo siguiente en la tabla 1.

Tabla 1. Porcentaje de los resultados en el ámbito social.

\begin{tabular}{|c|l|c|c|}
\hline $\mathbf{N}^{\circ}$ Criterios & \multicolumn{1}{|c|}{ Ámbito social } & SI & NO \\
\hline 1 & $\begin{array}{l}\text { Se promueve la cultura sobre desarrollo sostenible con } \\
\text { sus empleados a través de capacitaciones, } \\
\text { entrenamientos o campañas de concientización. }\end{array}$ & $100 \%$ & \\
\hline 2 & $\begin{array}{l}\text { La empresa estimula el desarrollo de prácticas } \\
\text { sostenibles entre sus empleados, fomentando así la } \\
\text { creación de un ambiente favorable laboral. }\end{array}$ & $100 \%$ & $\begin{array}{l}\text { La empresa posee algún código de conducta para la } \\
\text { selección de sus proveedores. }\end{array}$ \\
\hline 3 & $42,86 \%$ & $57,14 \%$ \\
\hline
\end{tabular}


OAI-PMH: http://www.indteca.com/ojs/index.php/Revista Scientific/oai

\begin{tabular}{|c|c|c|c|}
\hline $\mathbf{N}^{\circ}$ Criterios & Ámbito social & SI & NO \\
\hline 4 & $\begin{array}{l}\text { La empresa trabaja con proveedores que concienticen } \\
\text { el desarrollo sostenible. }\end{array}$ & $28,57 \%$ & $71,43 \%$ \\
\hline 5 & $\begin{array}{l}\text { La empresa informa a los clientes sobre prácticas } \\
\text { responsables que tienen con el medio ambiente o en lo } \\
\text { social. }\end{array}$ & $100 \%$ & \\
\hline 6 & $\begin{array}{l}\text { La empresa informa a sus clientes los productos } \\
\text { medioambientalmente responsables. }\end{array}$ & $100 \%$ & \\
\hline 7 & $\begin{array}{l}\text { La empresa se involucra con el desarrollo de la } \\
\text { comunidad. }\end{array}$ & $100 \%$ & \\
\hline 8 & $\begin{array}{l}\text { La empresa prioriza el bienestar de la comunidad } \\
\text { cercana a sus plantas procesadoras. }\end{array}$ & $57,14 \%$ & $42,86 \%$ \\
\hline & Promedio ámbito social & $78,57 \%$ & $21,43 \%$ \\
\hline
\end{tabular}

Fuente: Los Autores (2021).

Los resultados de las encuestas reflejaron que las empresas exportadoras de camarón de la localidad poseen una relación positiva con sus empleados y clientes al capacitar e incentivar a su personal sobre prácticas sostenibles, así mismo al informar a sus consumidores sobre la responsabilidad medioambiental de la organización.

En cuanto a la relación con sus proveedores, el $71,43 \%$ de la población evidencio el no conocer si posean practicas sustentables, mientras el otro $28,57 \%$ manifestó que estos están alineadas a sus mismos propósitos y objetivos. Respecto a su relación con la comunidad, el 57,14\% respondió afirmativamente al priorizar el bienestar de la comunidad cercana a las plantas procesadoras, al incorporar lo más frecuentemente, diversas medidas para mitigar cualquier daño que pueda incomodar a las comunidades cercanas; el otro 42,86\% fundamentó el cumplir con los requisitos básicos establecidos el código de conducta para el funcionamiento de la planta procesadora.

Para la segunda variable, se analizó la responsabilidad de las empresas desde el punto de vista ambiental, tomando en cuenta las nuevas estructuras tecnologías, la gestión ambiental y las certificaciones como criterios que impulsan el desarrollo sostenible de la empresa. En el ámbito económico se relacionó con los intereses de los accionistas en función de las estrategias 
sobre los ingresos generados para la organización, obteniendo lo expresado en la tabla 2.

Tabla 2. Porcentaje de los resultados en el ámbito medioambiental y económico.

\begin{tabular}{|c|l|c|c|}
\hline $\mathbf{N}^{\circ}$ Criterios & \multicolumn{1}{|c|}{ Ámbito medioambiental } & SI & NO \\
\hline 1 & $\begin{array}{l}\text { La empresa posee certificaciones ligadas al } \\
\text { desarrollo sostenible medio ambiental. }\end{array}$ & $85.71 \%$ & $14.29 \%$ \\
\hline 2 & $\begin{array}{l}\text { La empresa aplica planes enfocados a la } \\
\text { gestión ambiental. }\end{array}$ & $100 \%$ & - \\
\hline 3 & $\begin{array}{l}\text { La empresa aplica estructuras tecnológicas que } \\
\text { fomenten el desarrollo sostenible. }\end{array}$ & $100 \%$ & - \\
\hline \multicolumn{1}{|c|}{ Promedio ámbito medioambiental } & $\mathbf{9 5 , 2 4 \%}$ & $\mathbf{4 , 7 6 \%}$ \\
\hline $\mathbf{N}^{\circ}$ Criterios & \multicolumn{1}{|c|}{$\mathbf{S I}$} & $\mathbf{N O}$ \\
\hline 1 & $\begin{array}{l}\text { Las estrategias de negocios que involucran el } \\
\text { desarrollo sostenible han permitido incrementar } \\
\text { las utilidades o ingresos de la organización. }\end{array}$ & $100 \%$ & - \\
\hline & Promedio ámbito económico & $\mathbf{1 0 0 \%}$ & - \\
\hline
\end{tabular}

Fuente: Los Autores (2021).

Para la variable medioambiental, todas las empresas tienen un índice porcentual positivo ya que estas tienen un alto grado de responsabilidad, estas utilizan diferentes herramientas tecnológicas que le permiten un mayor desarrollo de su gestión ambiental.

En las certificaciones ligadas con el desarrollo sostenible el $85,71 \%$ posee una certificación que le permite mantener un elevado desarrollo y productividad manteniendo sus procesos de manera eficiente y sustentable mientras que $14,29 \%$ de las empresas mantiene certificaciones de ámbito internacional, pero están enfocadas de manera directa con el desarrollo sustentable de las mismas.

De acuerdo con la tabla anterior, todas las empresas camaroneras de la provincia de El Oro poseen estrategias de negociación que involucran el desarrollo sostenible, además, que las mismas han demostrado un incremento en los ingresos económicos de la organización después de su aplicación. 
En la tabla 3, se muestran los resultados generales de cada ámbito en función al desarrollo sostenible, así mismo un promedio porcentual de todas las variables, lo que permitió valorar la situación de toda la población de estudio.

Tabla 3. Porcentaje de los resultados totales de cada ámbito de estudio.

\begin{tabular}{|l|c|c|}
\hline \multirow{2}{*}{\multicolumn{1}{|c|}{ Ámbito }} & \multicolumn{2}{c|}{ Resultado } \\
\cline { 2 - 3 } & $78,57 \%$ & $21,43 \%$ \\
\hline Social & $95,24 \%$ & $4,76 \%$ \\
\hline Ambiental & $100 \%$ & - \\
\hline Económico & $273,81 \%$ & $26,19 \%$ \\
\hline Total & $\mathbf{9 1 , 2 7 \%}$ & $\mathbf{8 , 7 3}$ \\
\hline Promedio Total de los tres ámbitos & \multicolumn{2}{|}{} \\
\hline
\end{tabular}

Fuente: Los Autores (2021).

Con base a los resultados mostrados en la tabla anterior, las empresas camaroneras de la provincia de El Oro poseen un desempeño altamente favorable en cuanto a su desarrollo económico y ambiental representado como un $100 \%$ y un $95,24 \%$, es decir sus estrategias están enfocadas al desarrollo de nuevas tecnologías o gestiones ambientales como certificaciones o mejoramientos de procesos más eficientes y eficaces. En su variable social, el $78,57 \%$ del total de la población cumplió de manera favorable los criterios para considerarse como empresas socialmente responsables con sus empleados, clientes, proveedores y comunidad.

\section{Discusión}

La sostenibilidad para las empresas, según Sánchez (2012): representa una nueva forma de cambio y transición, significa una nueva manera de realizar negocios (producción, comercialización, promoción y publicidad, etc.), siempre tomando en cuenta la mejor manera de relacionarse con la sociedad, ampliar el negocio y progresar en un mercado competitivo donde todos puedan generar una rentabilidad sostenida a largo plazo. 
Guiados por el objetivo de la investigación se enuncian cinco estudios realizados por otros autores que evalúan el desempeño de la responsabilidad social y desarrollo sostenible en las organizaciones. Con esta exposición se pretendió obtener los principales beneficios y resultados de los estudios.

El primer caso expone una tesis realizada por Ocaña y Pantoja (2018): sobre la incidencia de la sostenibilidad empresarial en las empresas camaroneras de Taura. Evidenciaron que la falta de conocimiento por parte de las empresas del sector, sobre las practicas sustentables, impedían el desarrollo sostenible de las empresas con relación a sus partes interesadas. Así mismo destacan que, las pocas organizaciones que practicaban el desarrollo sustentable, obtienen diversos beneficios, tales como una mejor relación con las partes colaboradoras al proporcionar la posibilidad de expandir sus negocios a un ámbito internacional.

El segundo caso es un estudio elaborado por Ibarra, Casas y Olivas (2012): en el estado de Hermosillo, Sonora sobre las prácticas de responsabilidad social enfocadas al desarrollo sustentable. Manifestaron que el sector poseía un desempeño favorable con expectativas de mejora en cuanto al desarrollo sostenible local y organizacional. Se comprueba que, al poseer un comportamiento responsable, las empresas se ven beneficiadas de una imagen atrayente, para nuevos mercados, generando una rentabilidad sostenida a largo plazo.

El tercer caso analiza la responsabilidad social de las empresas exportadoras más destacadas del Ecuador. Mencionando a Alvarado y Rugel (2017): las organizaciones demostraron un nivel bajo-medio en conocimiento sobre desarrollo sustentable, aun así, afirman que las empresas exportadoras son socialmente responsables. Este se debe a que las empresas cumplen con los requisitos estipulados por el mercado nacional e internacional. Así mismo, concluyen que las empresas son conscientes que, al mejorar la gestión en prácticas sostenibles, la organización y sus colaboradores gozarán de 
mayores beneficios.

El cuarto caso es un estudio realizado por Bravo y Arroyo (2018): con el objetivo de concientizar a los diversos sectores productivos del Ecuador para reducir el impacto ambiental. Enfatizan la importancia de implementar el desarrollo sostenible para generar una mayor productividad en las industrias ecuatorianas, de esta manera se reduciría el uso de recursos que conlleven a la contaminación del medio ambiente y mejoraría la calidad de vida de la población ecuatoriana.

El quinto caso expone la gestión ambiental de las empresas públicas y privadas de la ciudad de Guayaquil. Parafraseando a Malavé y Fernández (2020): corroboraron el desconocimiento que poseen las organizaciones sobre las ventajas que posee el desarrollo sostenible en relación con las mismas y sus partes interesadas, es por tal que, una parte de la población es indiferente a trabajar de manera eficiente para mantener el cuidado del medioambiente. Además, identificaron que el trabajo conjunto con instituciones gubernamentales permite conseguir mejores resultados a las organizaciones.

Con base a lo expuesto anteriormente, se coincide que las organizaciones que emplean estrategias de desarrollo sostenible poseen resultados propicios, tales como: expansión de negocios, creación de una imagen atractiva para nuevos mercados, fortalecimiento de las relaciones con sus partes colaboradoras y rentabilidad sostenida a mediano y largo plazo.

Así mismo, se estableció que el desarrollo sostenible del sector camaronero exportador de la provincia de El Oro, representando como el $91,27 \%$ en la tabla 3 , gestionan de manera eficiente sus prácticas sostenibles (social, económico y ambiental) con relación a sus partes interesadas, convirtiéndolas en empresas altamente competitivas en el mercado nacional e internacional, al saber manifestar un correcto desarrollo sustentable organizacional.

No obstante, cabe destacar que, durante las encuestas se evidencio 
que cinco empresas no poseían un área específica que se encargara de gestionar el desarrollo sostenible, tan solo dos de ellas disponían de una. Al aclarar este punto, podemos deducir que, si las organizaciones asumieran un personal capacitado en la creación de estrategias y practicas sustentables, podrían volverse ser más competitivas debido a su crecimiento empresarial constante.

Señalando a Remacha (2017a): el desarrollo sostenible contribuye al generar empleo, crear entornos de trabajo seguros, mejorar la calidad de ambiente de trabajo de los empleados al promover hábitos saludables infundadas a través de capacitaciones, comunicar valores en sus consumidores, invertir en tecnologías que apoyen a la economía circular al minimizar el consumo de recursos y generación de residuos. Además de conocer los diversos beneficios que trae consigo el desarrollo sostenible en una organización también es importante conocer las pautas necesarias para implementar estrategias relacionadas al mismo.

Según Remacha (2017b): las empresas deben estar alineadas conjuntamente a los objetivos de desarrollo sostenible establecidos por la Organización de las Naciones Unidas (ONU); Al seguir una serie de pasos, tales como: entender, priorizar, establecer objetivos, integrar y comunicar, las empresas lograrán incorporar los objetivos de desarrollo sostenible de la ONU a sus estrategias corporativas.

Interpretando a Gill y Barcellos (2011): establecen que, para una correcta toma de decisiones en una organización, se deben desarrollar modelos de gestión en base a los tres pilares del desarrollo sostenible (social, económico y ambiental) tomando en cuenta los grupos de interés.

Así mismo, Schneider (2019b): determina que una estrategia de sostenibilidad debe estar alineada a los objetivos y visión de la organización, así mismo deben estar diseñadas en base a la integración de las partes interesadas identificadas en el análisis de materialidad de la organización 
Una técnica denominada Prieto-Sandoval, Jaca y Ormazabal (2017): "de la cuna a la cuna" (pág. 91); es una de las estrategias de sostenibilidad ambiental más reconocida según lo indica. Esta estrategia permite que los productos se elaboran para ser utilizados nuevamente después de atravesar diversos fases bilógicas o técnicas.

Para Alaña, Capa y Sotomayor (2017): una forma de alanzar el desarrollo sostenible es compaginando el crecimiento económico conjuntamente con la equidad social y la protección y administración del medio ambiente, manteniendo el desarrollo continuo de manera conjunta, eficaz y eficiente.

Algunos de los autores antes mencionados concuerdan que las estrategias de sostenibilidad deben estar enfocadas en los tres pilares del desarrollo sostenible, de igual forma deben estar alineadas para cumplir con los objetivos, políticas y responsabilidades sociales a largo plazo de la organización, logrando así una mejora e innovación continua que permita obtener una mayor ventaja frente a sus competidores.

Ante esto podemos evidenciar que algunas de las empresas exportadoras de camarón de la provincia de el Oro, tienen un desempeño considerable en cuanto al desarrollo sostenible. Igualmente, buscan implementar estrategias que mejoren el desarrollo y productividad de la organización, teniendo una mayor oportunidad en los mercados internacionales y frente a sus competidores, por ello se mantienen en constante evolución con la aplicación de herramientas tecnológicas que les permitan una mejora continua en sus procesos productivos.

\section{Conclusiones}

El estudio fue realizado con el propósito de conocer si las empresas exportadoras de camarón poseen un desarrollo sustentable apropiado, por tal para alcanzar el objetivo de la investigación se estudió a las organizaciones y 
sus actividades considerando los tres pilares que conforman el desarrollo sostenible y la satisfacción que generan en sus stakeholders.

Los criterios establecidos para valorar la situación en desarrollo sostenible de las empresas exportadoras de camarón de la provincia de El Oro, demostraron que el sector posee un desempeño altamente favorable, convirtiéndolas en organizaciones más eficientes y eficaces al saber manejar sus recursos y gestionar sus procesos para lograr una mayor productividad a corto y largo plazo.

Con el fin de profundizar el tema planteado, se corroboró la investigación con criterios de otros autores que analizaron estrategias y situaciones similares a la estudiada. Por consiguiente, se observó que las empresas que poseen un desarrollo sostenible mejoran sus relaciones con sus stakeholders, generan rentabilidades sostenidas a largo plazo, mejoran su imagen empresarial y aumentan la posibilidad de expandir sus negocios a nuevos mercados internacionales.

\section{Referencias}

Alaña, T., Capa, L., \& Sotomayor, J. (2017). Desarrollo sostenible y evolución de la legislación ambiental en las MIPYMES del Ecuador. Universidad y Sociedad, 9(1), 91-99, e-ISSN: 2218-3620. Recuperado de: https://rus.ucf.edu.cu/index.php/rus

Alvarado, S., \& Rugel, T. (2017). Responsabilidad Social de las Empresas. Caso de estudio Empresas Exportadoras del Ecuador. Milagro, Ecuador: Universidad Estatal de Milagro. Recuperado de: http://repositorio.unemi.edu.ec/handle/123456789/3756

BBVA (2014). Reinventar la empresa en la era digital. España: Turner; Banco Bilbao Vizcaya Argentaria, S.A.

Bravo, D., \& Arroyo, F. (2018). Innovación sustentable: un camino al desarrollo productivo del Ecuador. Innova Research Journal, 3(5), 
29-44, e-ISSN: 2477-9024. Recuperado de:

https://doi.org/10.33890/innova.v3.n5.2018.512

Contreras-Pacheco, O., Talero-Sarmiento, L., \& Escobar-Rodríguez, L. (2020).

Sostenibilidad, stakeholders y crisis de empresa: un análisis estructurado de percepciones. Suma de Negocios, 11(24), 64-72, eISSN: 2215-910X. Recuperado de:

http://doi.org/10.14349/sumneg/2020.V11.N24.A7

Freeman, R., \& McVea, J. (2001). A Stakeholder Approach to Strategic Management. SSRN Electronic Journal, (01-02). Recovered from: http://dx.doi.org/10.2139/ssrn.263511

Gill, A., \& Barcellos, L. (2011). Los desafíos para la sostenibilidad empresarial en el siglo XXI. Revista Galega de Economía, 20(2), 1-22, e-ISSN: 1132-2799. Recuperado de:

https://www.redalyc.org/articulo.oa?id=39121262007

Ibarra, L., Casas, E., \& Olivas, E. (2012). La Responsabilidad Social Empresarial: Una estrategia enfocada al desarrollo sustentable. Caso: Las ESR de Hermosillo, Sonora. Revista Iberoamericana de Contaduría, Economía y Administración, 1(1), 1-18, e-ISSN: 2007-9907. Recuperado de: https://www.ricea.org.mx/index.php/ricea/article/view/9 Malavé, E., \& Fernández, M. (2020). Gestión ambiental de las empresas públicas y privadas en la ciudad de Guayaquil - Ecuador y su incidencia en el desarrollo sostenible. Sinergias educativas, 1(5), 110, e-ISSN: 2661-6661. Recuperado de:

http://portal.amelica.org/ameli/jatsRepo/382/3821581012/index.html

Manfredi, L. C., \& González, É. (2017). Autopartes del Caribe. Negociando estratégicamente. Estudios Gerenciales, 33(144), 309-316, e-ISSN: 0123-5923. Recuperado de:

http://dx.doi.org/10.1016/j.estger.2017.07.003

Mora-Córdova, D., Lituma-Loja, A., \& González-Illescas, M. (2020). Las 
certificaciones como estrategia para la competitividad de las empresas exportadoras. Innova Research Journal, 5(2), 113-132, eISSN: 2477-9024. Recuperado de:

https://doi.org/10.33890/innova.v5.n2.2020.1274

Ocaña, H., \& Pantoja, N. (2018). Análisis de las prácticas de la responsabilidad social empresarial y su incidencia en la sostenibilidad empresarial en el sector camaronero de la parroquia Taura, cantón Naranjal, provincia del Guaya. Guayaquil, Ecuador: Universidad de Guayaquil. Recuperado de:

http://repositorio.ug.edu.ec/handle/redug/34734

OIT (2007a,b). Informe VI. La promoción de empresas sostenibles. Primera edición, ISBN: 978-92-2-318143-7; ISSN: 0251-3226. Ginebra, Suiza: Oficina Internacional del Trabajo.

OMC (2011). Aprovechamiento del comercio para el desarrollo sostenible y una economía verde. ISBN: 978-92-870-3808-1. Ginebra, Suiza: Organización Mundial del Comercio.

Prieto-Sandoval, V., Jaca, C., \& Ormazabal, M. (2017). Economía Circular: Relación con la evolución del concepto de sostenibilidad y estrategias para su implementación. Memoria Investigaciones en Ingeniería, (15), 85-95, e-ISSN: 2301-1106. Recuperado de: https://hdl.handle.net/10171/53653

Remacha, M. (2017a,b). Empresa y objetivos de desarrollo sostenible. Cuadernos de la Cátedra CaixaBank de Responsabilidad Social Corporativa, Nº 34. España: IESE Business School; Universidad de Navarra.

Sánchez, N. (2012). La sostenibilidad en el sector empresarial: importancia de los distintos grupos de interés en el proceso de cambio a sostenibilidad en el sector empresarial. Barcelona, España: Universitat Politècnica de Catalunya. Recuperado de: 
Instituto Internacional de Investigación y Desarrollo Tecnológico Educativo INDTEC, C.A.

DOI: https://doi.org/10.29394/Scientific.issn.2542-2987.2021.6.22.11.210-230

OAl-PMH: http://www.indteca.com/ojs/index.php/Revista Scientific/oai

Artículo Original / Original Article



http://hdl.handle.net/2099.1/18820

Schneider, H. (2019a,b). Guía para integrar la sostenibilidad en las empresas. El Salvador: Departamento de Estado de los Estados Unidos; Programa Centroamérica Resiliente (ResCA); The Nature Conservancy.

UNSDG (2018). Desafíos y estrategias para el desarrollo sostenible en américa latina y el caribe. Nueva York, Estados Unidos: Grupo de Desarrollo Sostenible de las Naciones Unidas para América Latina y el Caribe, Panamá.

Zarta, P. (2018). La sustentabilidad o sostenibilidad: Un concepto poderoso para la humanidad. Tabula Rasa, (28), 409-423, e-ISSN: 1794-2489. Recuperado de: https://doi.org/10.25058/20112742.n28.18 


\section{Aylin Madelaine Honores Gómez}

e-mail: ahonores3@utmachala.edu.ec

Nacida en la ciudad de Machala, provincia de El Oro en

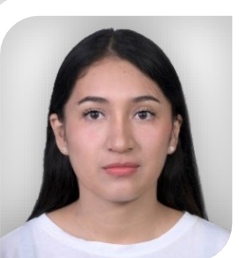

Ecuador, el 30 de agosto del año 1996. Egresada de la carrera de Comercio Internacional de la Universidad Técnica de Machala (UTMACH); he participado en diversos congresos en base a la carrera dentro del país; posee un nivel de inglés intermedio-alto que me ha permitido participar en programas de intercambio cultural y laboral con Estados Unidos; he realizado cursos en base a diseño gráfico y pastelería creativa; participé en diversos concursos de diseño gráfico de los cuales soy ganadora; actualmente me dedico a la pastelería creativa. 


\section{Byron Alexander Chavarría Sangurima}

email: bchavarri1@utmachala.edu.ec

Nacido en la provincia de El Oro, Ecuador, el 22 de

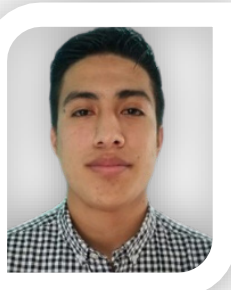

octubre del año 1998. Egresado de la carrera Comercio Internacional de la Universidad Técnica de Machala (UTMACH); he participado en ponencias universitarias; asistido a congresos nacionales e internacionales sobre comercio exterior y negocios internacionales, marketing y administración; he participado en voluntariados comunitarios; tengo un nivel de inglés intermedio; he participado en diversos puestos laborales lo que me ha permitido desarrollar diferentes habilidades técnicas y sociales. 


\section{Sandra Sayonara Solórzano Solórzano \\ e-mail: ssolorzano@utmachala.edu.ec}

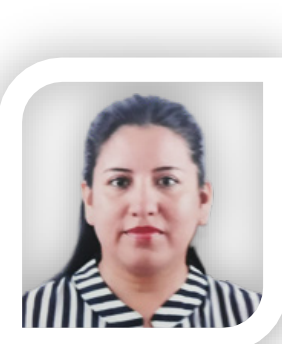

Nacida en la provincia de El Oro, Ecuador, el 1 de septiembre del año 1975. Docente-investigadora de la Universidad Técnica de Machala (UTMACH); Ingeniera en Comercio Internacional con título obtenido en la Universidad Técnica de Machala; Magister en Administración y Dirección de Empresas, con título obtenido en la Universidad Tecnológica Empresarial de Guayaquil (UTEG); coordinadora de la Carrera de Comercio Exterior; Ocho años de experiencia en docencia universitaria; he publicado libros y artículos científicos en la temática del comercio exterior; también he participado en ponencias nacionales e internacionales; Miembro de diferentes grupos y proyectos de Investigación.

El contenido de este manuscrito se difunde bajo una Licencia de Creative Commons ReconocimientoNoComercial-Compartirlgual 4.0 Internacional 